\title{
APLICAÇÃO DA METODOLOGIA DMAIC PARA AUMENTO DE PRODUTIVIDADE INDUSTRIAL
}

\section{DMAIC METHODOLOGY APPLICATION FOR PRODUCTIVITY INCREASE}

\author{
Welington Martins, Andréia de Menezes Olivo \\ Universidade do Oeste Paulista - UNOESTE, Faculdade de Engenharias e Arquitetura \\ e Urbanismo, Presidente Prudente, SP. \\ E-mail: well.limartins@gmail.com
}

RESUMO - O presente trabalho baseia-se na aplicação da metodologia DMAIC para o aumento da produtividade do processo produtivo de emulsificantes de uma empresa do setor alimentício. A metodologia adotada é de natureza quantitativa e exploratória tendo como procedimento técnico a pesquisa ação. A coleta de dados sucedeu-se de forma descritiva considerando as operações realizadas no setor produtivo e o tempo despendido para a realização de cada tarefa. Os dados coletados foram compilados em uma planilha com o auxílio de um software estatístico, onde foram analisados através de gráficos para obtenção do cenário real de produtividade do setor, assim como as causas impactantes geradoras de tempo improdutivo. A partir da análise dos dados e a aplicação das etapas estruturadas do método, desenvolveu-se o plano de ação para trabalhar a melhoria proposta. Os resultados obtidos corroborou a eficácia da metodologia DMAIC, pois os resultados apresentados foram satisfatórios e significativos com a obtenção do aumento da produtividade em $10 \%$.

Palavras-chave: DMAIC; Produtividade; Processo; Melhoria Contínua; Metodologia.

ABSTRACT - This present work is based on the application of the DMAIC methodology for the productivity increase of the production process of emulsifier in a food company. The methodology has quantitative and exploratory nature having as technical procedure action research. The data collection occurred is descriptively considering the operations in the productive sector and the time taken to perform each task. The data was compiled in a spreadsheet with the assistance of statistical software, which was analyzed using charts to obtain the real scenario sector's productivity, as well as the causes downtime generating impactful. From the analyses of the data and the application of structured steps of the method, developed the action plan to work to improve the proposal. The results corroborated the

Recebido em: 25/08/2016 Revisado em: 26/08/2016 Aprovado em: 02/09/2016 effectiveness of DMAIC because the results were satisfactory and significant with achievement of the productivity increase in $10 \%$.

Keywords: DMAIC; Productivity; Process; Continuo Improvement; Methodology. 


\section{INTRODUÇÃO}

O principal objetivo de uma empresa é atender a demanda de seus clientes, no prazo acordado, com qualidade especificada, preço competitivo, produzindo com baixo custo e finalmente a obtenção do lucro desejado. Porém, para atingimento de todos esses atributos faz-se necessário processos robustos e sustentáveis. Para isso, o uso de ferramentas, métodos e metodologias são indispensáveis para garantir a eficiência e eficácia dos resultados. Portanto, o programa Seis Sigmas vêm sendo disseminado pelas grandes corporações mundiais, sendo a metodologia DMAIC, cerne desse programa, utilizada para a resolução de problemas de processos, gerando resultados extremamente significativos para as companhias. Dentre os maiores benefícios na utilização simultânea e integrada das ações para a qualidade na busca da melhoria e dos projetos DMAIC pode-se citar: alinhamento dos processos, realinhamento cultural, redução dos desperdícios, redução de falhas, melhoria da produtividade, melhoria na qualidade, atendimento as necessidades e as expectativas dos clientes (RODRIGUES, 2014).

Atualmente as companhias encontram-se inseridas em um cenário de grande competitividade do mercado em virtude da globalização. Desta forma, as empresas tentam buscar novas formas de tornar-se competitivas para garantir a sua permanência no mercado. Todavia, para o alcance desse objetivo requer que as empresas adotem o controle e a eliminação de desperdícios como prioridade (OLIVEIRA, 2003).

Diante do grande potencial de ganhos de produtividade existentes nos processos fabris, é de fundamental importância conhecer as causas que impactam na eficiência do processo tornando a empresa menos produtiva. $\mathrm{O}$ presente trabalho vem demonstrar os ganhos de produtividade concebidos através da aplicação da ferramenta DMAIC em uma empresa alimentícia localizada na região de Presidente Prudente. E para o meio ambiente, o trabalho trará benefícios a abstenção de perdas de recursos, para a operação o ganho de eficiência no trabalho e, por fim para a academia, o conhecimento adquirido pela prática da metodologia aplicada.

\section{SEIS SIGMA}

O conceito Seis Sigma foi criado dentro da empresa Motorola na década de 1980, e logo depois difundido para outras empresas de classe mundial devido os ganhos obtidos pela sua implementação. $\mathrm{O}$ programa baseia-se na adoção de ferramentas da qualidade e pensamento estatísticos, na análise sistêmica de variabilidade de processo e também a integração do gerenciamento por processos e diretrizes, foco nos clientes, nos processo 
críticos e resultados da empresa (MONTEIRO; PALADINI, 2012).

Segundo Pande (2013), "O Modelo MAIC (Medir, Analisar, Melhorar, Controlar) foi desenvolvida inicialmente na Motorola como uma evolução do ciclo PDCA e depois adotada pela GE como DMAIC, onde foi inserido a fase Definir. Esse método passou a ser a base operacional da ruptura Seis Sigma para essas empresas, sendo fundamental para o sucesso que alcançaram".

DMAIC

O DMAIC é uma metodologia estruturada para a análise e solução de problemas e oportunidade tendo como foco a identificação da causa raiz encontrando soluções de melhoria duradouras. Trata-se de um passo a passo para a solução definitiva do problema (NICOLETTI, 2011).

Os pontos fortes do DMAIC estão evidentes, afinal não há conflitos entre o DMAIC e o PDCA e, sim, um complemento, de modo que ambos podem ser utilizados em uma mesma empresa. O DMAIC têm um roteiro detalhado e maior número de ferramentas analíticas, pode e deve complementar o PDCA, à medida que evoluem o sistema de gestão e o nível de capacidade das pessoas da organização (WERKEMA, 2013).

O DMAIC fornece além de orientação, as ferramentas a serem executadas com o objetivo de planejar, implementar e padronizar processos de melhoria altamente significativos, que são em geral associados a ganho financeiro e/ou fidelização do cliente. O DMAIC consolida a utilização de raciocínio lógico formal. A utilização do DMAIC visa auxiliar a organização e a equipe de projeto na análise dos dados e informações acerca do problema (CAUCHINCK; FERREIRA, 2011).

A metodologia DMAIC é um aspecto muito importante, já que uma organização pode obter importantes benefícios ao institucionalizar a empresa ao DMAIC para a resolução de problemas, eliminando, desta forma, os problemas decorrentes (GUPTA; SRI, 2012).

O modelo DMAIC focaliza na busca de soluções de problemas e oportunidades por meio de decisões baseadas em dados, fundamentada em dados quantitativos e não em intuição (CERVO, 2007).

Para Chamon (2008) o modelo DMAIC concentra várias outras ferramentas que auxilia na definição, análise, identificação de melhorias nos processos em que a variabilidade esteja presente, eliminando ou reduzindo a ocorrência de defeitos no processo, neste momento é considerado tanto o cliente final externo quanto o cliente interno do processo.

O DMAIC permite uma análise imparcial e direta do problema, evitando assim, que concentremos esforços em sentido oposto ao da solução do problema. Usando a metodologia passo a passo, garantimos o foco no problema original, 
aumentando em muito a probabilidade de sucesso das ações (NICOLETTI, 2011).

O DMAIC apresenta alguns pontos fortes como o foco no ganho financeiro, a utilização do DMAIC é um diferencial, pois é a utilização de uma metodologia estruturada para a resolução dos problemas. Como pontos negativos os autores também citam que um excesso de rigor na utilização do DMAIC pode acabar por amarrar o processo de resolução de problemas, como também a hierarquia apesar de potencializar o processo acaba por excluir algumas pessoas que estão em contato direto com o processo que poderiam ajudar com ideias inovadoras na resolução dos problemas (FERNANDES; RAMOS, 2006).

\section{METODOLOGIA}

A primeira fase do projeto é denominada Definir (Define), nela foram definidos os requisitos do clientes (VOC Voice of Customer) e traduzidos em CTQ (Critical To Quality), ou seja, Características Críticas para a Qualidade do produto ou serviço. Nessa etapa identificou-se os cliente como sendo os membros de nível operacional e tático que atuam diretamente no setor, ou seja, operadores e líderes de operação. Para capturar as necessidades do cliente aliada à estratégia do negócio, utilizou-se de entrevistas não estruturadas e para definição do VOC utilizou-se de uma matriz QFD (Quality Funtion Deployment Desdobramento da Função Qualidade).

Após a identificação e definição do problema, seguiu-se para a próxima etapa denominada contrato do projeto. Elaborouse um documento contendo um resumo do projeto com descrição do problema, quando e em que condições ocorrem o problema, onde ocorre, qual a extensão e o impacto.

Para finalizar a fase Define, definiu-se - mapa do processo utilizando-se da ferramenta SIPOC, onde foram identificados os Suppliers, Inputs, Outputs e Customers do processo estudado.

A próxima fase da metodologia DMAIC é denominada Medir (Measure). Nesta fase, responde-se a pergunta sobre como o processo é medido e como é executado. Carvalho e Paladini (2012) definem essa fase como o desenho do processo e os subprocessos que relacionamse com as CTQs, definindo as entradas e saída, tão como relacionar o $Y=f(X)$. Na etapa, seguinte definiu-se $\circ \mathrm{Y}$ do projeto através da ferramenta de mapeamento de processo. Depois, mensurou-se os padrões de desempenho, tais como a definição da unidade utilizada para medir o $Y$ do projeto, as características críticas, padrão de desempenho atual e defeitos do processo que não estavam de acordo com os padrões requeridos. Após definido o problema do processo (Y), estabeleceu-se o plano de 
coleta de dados, ou seja, o que medir como medir e quem medir.

Utilizando-se da ferramenta Diagrama de Causa e Efeito, elencou-se as possíveis causas que impactam no problema, ou seja, perdas de tempo no processo. Em seguida, compilou-se os dados de apontamento e utilizando-se de um software estatístico gerando-se um gráfico sumário básico.

A fase seguinte é denominada Analisar (Analyse), com o uso da ferramenta Gráfico de Pareto, identificaram-se as causas de maiores impactos por perda de horas, sendo essas: Troca de produto, Falta de MP e Aguardando PE. Na primeira etapa dessa fase, utilizando-se do um diagrama de causa efeito, identificou-se a lista das principais causas (Xs) que impactavam por perda de horas $(\mathrm{Y})$ pelas três maiores causas citadas acima. Na sequência, utilizando-se de uma matriz QFD priorizou-se os Xs vitais, ou seja, os que tinha impactos mais significativos.

$\mathrm{Na}$ fase Melhorar (Improve), desenvolveu-se o plano de ação para atacar cada $X$ vital para o processo. E por fim, na fase Controlar (Control), desenvolveu-se o plano de controle.

\section{RESULTADOS E CONCLUSÕES}

O projeto foi desenvolvido com 0 objetivo de eliminar ou diminuir causas que contribuem para o desperdício de tempo, evitando-se assim períodos improdutivo no setor produtivo, pois com isso, otimiza-se o processo e há maior proveito dos recursos como mão-de-obra e energia. Além de propiciar o aumento de capacidade produtiva, criando-se possibilidade para absorver novas demandas.

Todas essas causas impactantes no processo estudado, estão relacionadas na Figura 01, assim como suas respectivas porcentagem de horas perdidas. No entanto, priorizou-se três maiores causas, a troca de produto, aguardando PE (o PE trata-se da primeira fase do processo onde são produzidos os intermediários que são destinados à finalização em pó no setor estudado) e falta de MPs, as quais somadas representaram 35\% do tempo improdutivo. 


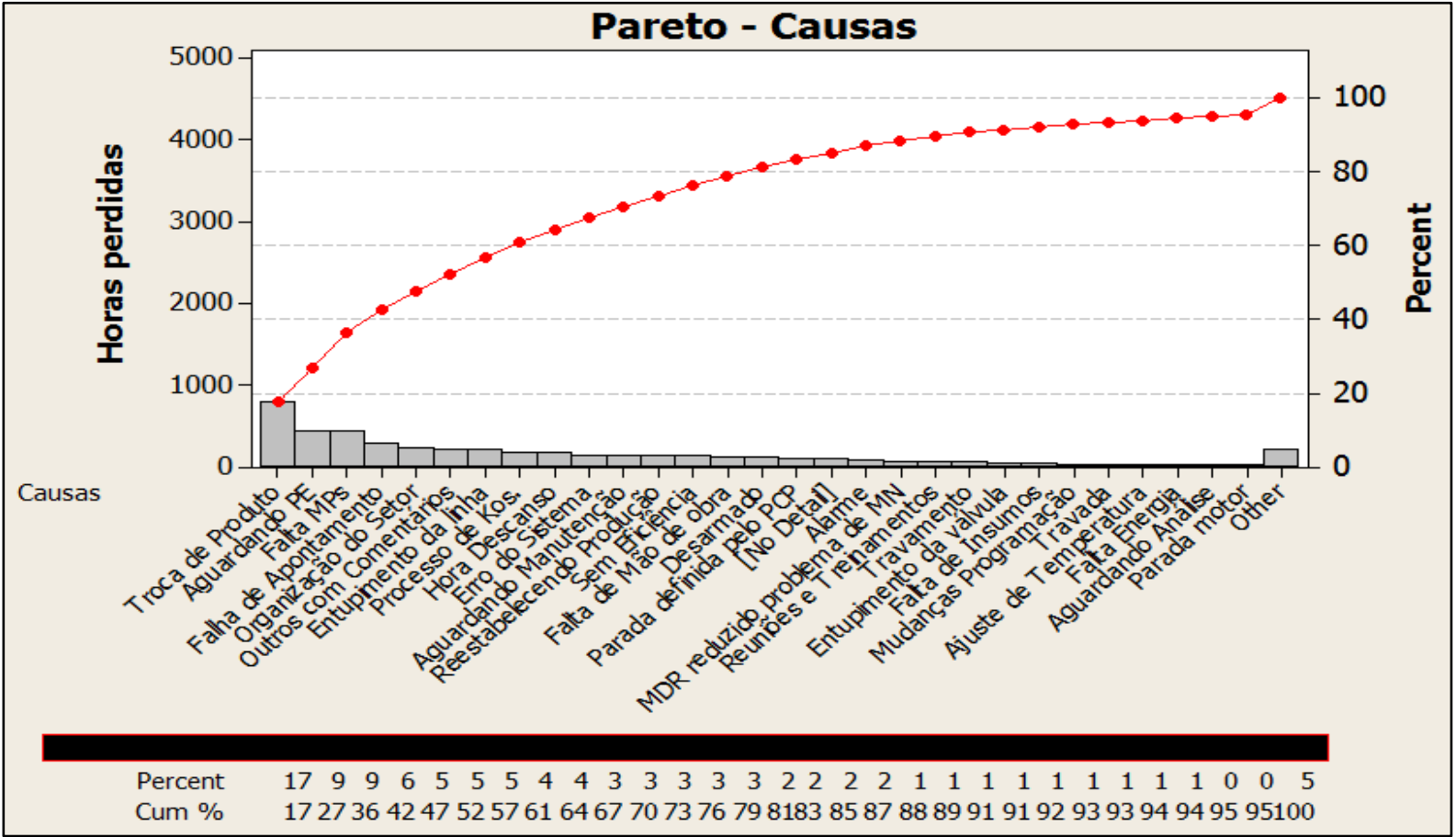

Figura 01. Relação das horas perdidas durante o turno de trabalho pelas causas que as ocasionam Fonte: dados trabalhados pelo autor.

Tabela 01. Comparativo entre cenário anterior e posterior à aplicação da metodologia.

\begin{tabular}{|l|c|c|c|}
\cline { 3 - 4 } \multicolumn{1}{c|}{} & \multicolumn{3}{c|}{ Horas perdidas } \\
\cline { 2 - 4 } \multicolumn{1}{c|}{} & $2013 / 2014$ & $2015 / 2016$ & Redução em \% \\
\hline Troca de produto & & & \\
\cline { 1 - 3 } Aguardando PE & 68,2 horas & 49,4 horas & $27,6 \%$ \\
\hline Falta de MP & & & $* * * * *$ \\
\hline Demais causas & 120 horas & $* * * * *$ & $10 \%$ \\
\hline \multicolumn{1}{|c|}{ Total } & 188,2 horas & $* * * * *$ & 10 \\
\hline
\end{tabular}

Fonte: Dados trabalhados pelo autor.

Conforme observa-se na Tabela 1, a linha base (anos 2013/2014) comparada com os resultados atingidos em 2015/2016, conclui-se que houve redução significativa de horas perdidas, tão somente pelas três causas principais, que passou de uma média de 68,2 horas/mês para 49,4 horas/mês, ou seja, houve uma redução de $27,6 \%$. Já em relação a linha base total de horas paradas, a redução significou $10 \%$.

Outro fato importante observado foi a redução de variabilidade do processo, ou seja, demonstrando-se mais mais estável e controlável. Essa conclusão foi verificada com a redução do desvio padrão entre a linha base que era de 55,27 horas passando a ser 40,41 horas pós melhorias.

\section{CONCLUSÃO}

O trabalho apresentado abordou a aplicação dos conceitos e utilização da metodologia DMAIC no processo produtivo de uma indústria de alimentos situada na região de Presidente Prudente com o intuito 
de reduzir o desperdiço de tempo. Pode-se afirmar que a aplicação da metodologia no processo, assim como a utilização das ferramentas da qualidade trouxeram resultados satisfatório e contribuiram para melhoria sistêmica do processo estudado.

Com a aplicação da metodologia, observou-se que houve diminuição de horas perdidas pelas causas maiores, ou seja, troca de produto, aguardando PE e falta de MP. Comparando-se com a linha base o ganho foi de $27,6 \%$ e se, comparado com o total de todas as causas, o ganho foi de $10 \%$, ou seja, com a diminuição de horas paradas ganhouse em produtividade, ampliação da capabilidade, redução de custo por quilograma de produto e melhor utilização do recurso humano.

Finalizando, conclui-se que o programa Seis Sigma e a metodologia DMAIC pode ser visto como uma importante arma de competitividade, pois permite a melhoria de resultados de processo bem como a redução de variabilidade do mesmo, permitindo reduzir as perdas de processo e garantindo o máximo de lucratividade para a empresa.

\section{REFERÊNCIAS}

CARVALHO, M. M. de; PALADINI, E. P. (Coords.). Gestão da qualidade: Vivências: Revista Eletrônica de Extensão da URI. Vivências. Vol. 11, N.20: p.177-189. 2. ed. Rio de Janeiro: Elsevier, 2012.
CAUCHINCK, P. A.; FERREIRA, M. C. V. Projeto de produto. 1. ed. Rio de Janeiro: Elsevier, 2011.

CERVO, A. L. Metodologia científica. 6. Ed. São Paulo: Pearson Prentice Hall, 2007.

CHAMON, E.M.Q.O. Gestão integrada de organizações: seis sigma. 1. ed. Rio de Janeiro: Brasporte, 2008.

FERNANDES, P. M. P.; RAMOS, A. W. Considerações sobre a integração do lean thinking com o seis sigma. In: ENEGEP, 26., 2006, Fortaleza. Anais... Fortaleza, CE: UNIFor, 2006.

GUPTA, P.; SRI, A. Seis sigma: enfoque no objetivo para alcançar rápidas melhorias. 1. ed. Cidade do Porto - PT: Vida Econômica, 2012.

MONTEIRO, C.; PALADINI, E.P. Gestão da qualidade. 2. ed. Rio de Janeiro: Campus, 2012.

NICOLETTI, A. Introdução ao lean seis sigma: coleção de melhoria contínua. 1. ed. São Paulo: Clube de Autores, 2011.

OLIVEIRA, J. O. Gestão da qualidade: tópicos avançados. São Paulo: Thomson, 2003.

PANDE, P. S. Estratégia seis sigma: estratégia + experiência operacional = desempenho superior. 1. ed. São Paulo: Bookman, 2013.

RODRIGUES, M. Ações para a qualidade: gestão estratégica e integrada para melhoria dos processos na busca da qualidade e competitividade. 5. ed. Rio de Janeiro: Elsevier, 2014.

WERKEMA, C. Métodos PDCA e DMAIC e suas Ferramentas Analíticas. Rio de Janeiro: Elsevier, 2013. 